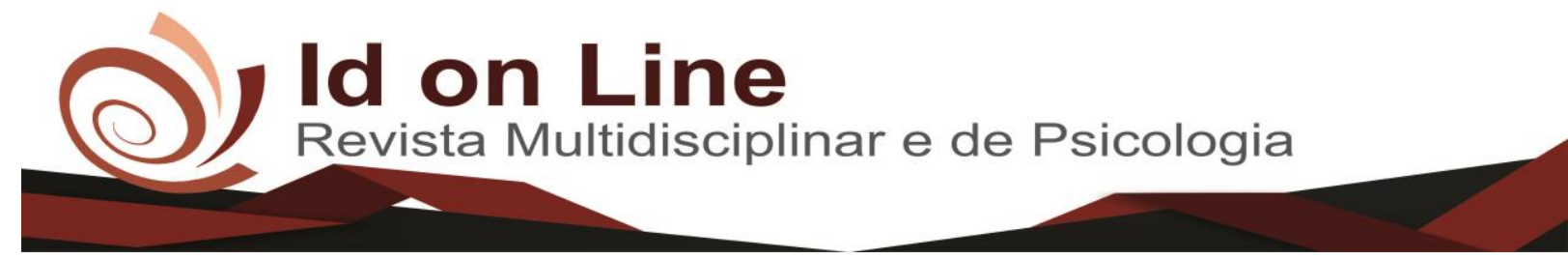

Artigo

\title{
Percepção dos Pacientes Hipertensos sobre o Acolhimento na Atenção Primária à Saúde
}

\author{
José Adelmo da Silva Filho ${ }^{1}$; Milana Correia Cunha ${ }^{2}$; Rosely Leyliane dos Santos ${ }^{3}$; \\ Itala Alencar Braga Victor ${ }^{4}$; Roberta Peixoto Vieira ${ }^{5}$; Ingrid Mikaela Moreira de Oliveira ${ }^{6}$
}

\begin{abstract}
Resumo: O estudo objetivou analisar a percepção de pacientes hipertensos sobre o acolhimento prestado por profissionais da saúde na atenção primária. Estudo descritivo, exploratório com abordagem qualitativa. Participaram do estudo 20 hipertensos cadastrados em uma Unidade Básica de Saúde do município de AcopiaraCeará. Utilizou-se como meio de coleta a entrevista semiestruturada, sendo a análise de dados através do discurso do sujeito coletivo. O estudo foi aprovado pelo Comitê de Ética em Pesquisa da Universidade Regional do Cariri pelo parecer $\mathrm{n}^{\circ}$ 1.937.176. A partir dos resultados encontrados percebeu-se que o acolhimento, assim como também as necessidades em saúde são superficiais, limitando o comparecimento dos pacientes à unidade de saúde, precisando vir a ser desenvolvidas ações por parte dos profissionais para facilitar o acolhimento a esses pacientes através do vínculo entre os envolvidos. Faz-se necessário ações de educação em saúde para conscientizar esses pacientes hipertensos quanto ao seu estado de saúde.
\end{abstract}

Descritores: Atenção Primária em Saúde. Tecnologias em Saúde. Acolhimento. Hipertensão Arterial Sistêmica.

\section{Perception of Hypertensive Patients on Reception in Primary Health Care}

\begin{abstract}
The study aimed to analyze the perception of hypertensive patients about the care provided by health professionals in primary care. Descriptive, exploratory study with a qualitative approach. Twenty hypertensive patients enrolled in a Basic Health Unit in the city of Acopiara-Ceará participated in the study. The semistructured interview was used as a means of collecting, and data analysis was done through the discourse of the collective subject. The study was approved by the Research Ethics Committee of the Regional University of Cariri for opinion No. 1,937,176. From the results found, it was noticed that the reception, as well as the health needs are superficial, limiting the attendance of the patients to the health unit, needing to be developed actions by the professionals to facilitate the reception to these patients through the link between those involved. It is necessary to carry out health education actions to raise awareness among these hypertensive patients about their state of health.
\end{abstract}

Keywords: Primary Health Care. Health Technologies. Reception. Systemic Arterial Hypertension.

\footnotetext{
${ }^{1}$ Enfermeiro. Esp. em Saúde Mental. Residente em Saúde Mental Coletiva pela ESP/CE. adelmof12@gmail.com

${ }^{2}$ Enfermeira graduada pela Universidade Regional do Cariri - URCA. milanacorreia@ hotmail.com

${ }^{3}$ Enfermeira. Doutoranda em Enfermagem (UFC). Mestre em Enfermagem (URCA). Especialista em Assistência e Gestão em Saúde da Família (FJN). Docente do Departamento de Enfermagem da URCA. rosely.enfa@ yahoo.com.br

${ }^{4}$ Enfermeira. Especialista em Urgência e Emergência (FVS). Docente do Departamento de Enfermagem da URCA e FVS. italaalencar@hotmail.com

${ }^{5}$ Enfermeira. Mestre em Saúde da Criança e do Adolescente (UECE). Especialista em Assistência e Gestão em Saúde da Família (FJN). Docente do Departamento de Enfermagem da URCA e FVS roberta.peixotovieira@gmail.com

${ }^{6}$ Enfermeira. Mestre em Bioprospecção Molecular (URCA). Especialista em Enfermagem no Cuidado Pré-Natal (UNIFESP). Docente do Departamento de Enfermagem da URCAingrid_lattes@hotmail.com
} 


\section{Introdução}

A Atenção Primária em Saúde (APS) apresenta aspectos distintos e interdependentes: é utilizada como estratégia de organização e reorganização dos sistemas de saúde, representando o primeiro nível de atenção à saúde e o modelo de mudanças na prática clínica-assistencial (OLIVEIRA; PEREIRA, 2013).

No Brasil, o modelo de APS é denominado de Atenção Básica em Saúde (ABS) que desenvolve ações voltadas para a saúde como a promoção e a prevenção, abrangendo tratamento e a manutenção da saúde. Essa prática se desenvolve por meio do exercício de cuidado e gestão democrática ou participativa, mas que estejam direcionadas as populações de territórios definidos. Para isso, utiliza-se de tecnologias de saúde voltadas ao cuidado que auxiliam no manejo das demandas e necessidades de saúde presentes no seu território (BRASIL, 2017).

Após a implantação da Atenção Básica necessitava de inovações que suprisse o sistema vigente, nesse período surge a Estratégia Saúde da Família (ESF) priorizando ações desenvolvidas, de forma integral e continuada estruturando-se com base no reconhecimento das necessidades da população, estabelecendo vínculos entre os usuários dos serviços e os profissionais de saúde (OLIVEIRA; PEREIRA, 2013).

Com isto, para facilitar o acesso nos serviços de saúde e o desempenho das equipes multiprofissionais, a Política Nacional de Atenção Básica (PNAB) através da portaria $n^{\circ}$ Portaria $\mathrm{N}^{\mathrm{o}} 2.436$, de 21 de setembro de 2017, estabeleceu atribuições a todos os profissionais e dentre estas, participar do acolhimento aos usuários realizando a escuta qualificada e a identificação das necessidades de intervenções voltadas ao atendimento humanizado proporcionando o atendimento das suas necessidades (OLVIEIRA et al., 2015).

Nessa perspectiva, o acolhimento é, portanto, centrado no usuário e para que isso ocorra é necessária uma reorganização do processo de trabalho, quebrando o modelo biomédico e implicando para atuação da equipe multiprofissional, que é responsável por ouvir o usuário e comprometer-se com sua demanda de saúde (GUIMARÃES et al., 2017).

A relação entre profissionais da saúde e usuário não deve estar centrada em procedimentos, normas e prescrições, mas direcionada também para a educação em saúde. O uso das tecnologias é necessário para a resolução de situações que levam os usuários a 
procurarem as unidades de saúde, abrangendo os mesmos de forma holística (SANTOS; NERY; MATUMOTO, 2013).

Nessa perspectiva, o acolhimento destaca-se como uma tecnologia leve em saúde que organiza o processo de cuidado da unidade local para a realização da avaliação do estado de saúde e classificação de risco, dependendo essencialmente da interação entre profissionais e usuários. O profissional de saúde precisa estar apto para acolher, resolver, responsabilizar e tornar autônoma a assistência em saúde (LIMA; MOREIRA; JORGE, 2013).

Nas Unidades Básicas de Saúde (UBS), o acolhimento, como o processo que permeia a abordagem ao usuário; também é definido como um espaço específico onde se realiza a primeira escuta e se identifica a necessidade do paciente, orientando e direcionando ao local mais adequado para resolução do seu problema (BRASIL, 2013).

O acolhimento e a escuta qualificada estão incluídos nos objetivos da Política Nacional de Humanização (PNH) da Atenção e Gestão do Sistema Único de Saúde (SUS) e podem subsidiar as práticas dos profissionais de saúde para o atendimento das necessidades, dos usuários, no contexto do SUS.

Nesse sentido, a atenção à saúde no cotidiano da ESF, tem-se o atendimento de pacientes hipertensos como uma demanda representativa e que exige atenção quanto ao acolhimento. Isso se deve ao fato de que a Hipertensão Arterial Sistêmica (HAS) ser uma doença de alta prevalência, com perda importante da qualidade de vida, o que torna de grande eficácia a detecção precoce, necessitando mudanças no estilo de vida por parte dos pacientes (BARRETO et al., 2013).

A Hipertensão arterial chega a atingir 32,5\% pessoas no Brasil, correspondendo a 36 milhões de indivíduos adultos, sendo que desses mais de $60 \%$ são idosos, contribuindo direta ou indiretamente com $50 \%$ das morbimortalidades por doenças cardiovasculares (MALACHIAS et al. 2016).

A HAS apresenta características crônicas e silenciosas dificultando a percepção dos sujeitos vulneráveis a esse problema, comprometendo a qualidade de vida das pessoas, tendo como consequência altas taxas de morbimortalidade por ser um dos principais fatores de risco para o desenvolvimento de doenças cardiovasculares (OLOWE; ROSS, 2017).

A finalidade do acompanhamento ao usuário com HAS, como em qualquer outra doença crônica não transmissível, é conseguir o controle da doença e prevenir o desenvolvimento de 
complicações secundárias. Para tanto, é necessário o envolvimento de uma abordagem multipartidária, abrangendo a equipe de saúde e o usuário (OLOWE; ROSS, 2017).

Em meio a essa realidade, o acolhimento e o vínculo no cuidado às pessoas com HAS facilita a continuidade do tratamento, por meio do recebimento de informações adequadas por parte dos profissionais de saúde, agendamento de consultas e garantia de retorno dos usuários (LIMA; MOREIRA; JORGE, 2013).

Nesse cenário, o presente estudo foi conduzido a partir das seguintes perguntas norteadoras: os pacientes percebem o que é acolhimento? Quais os motivos que os levam a procurar a unidade de saúde? Suas necessidades em saúde são atendidas? Essas perguntas conduziram a construção do objeto desse estudo quando o mesmo se propôs a analisar a percepção de pacientes hipertensos sobre o acolhimento prestado por profissionais da saúde na atenção primária.

\section{Percurso Metodológico}

Trata-se de um estudo descritivo, exploratório com abordagem qualitativa. O estudo foi realizado em uma Unidade Básica de Saúde do Município de Acopiara-Ceará. A UBS realizava seus atendimentos na área desde 2010, divide-se em oito micro áreas e abrange uma totalidade de 1.024 famílias. Segundo os dados do Cadastro Nacional dos Estabelecimentos de Saúde (CNES), em dezembro de 2015, o município apresentava 1.483 hipertensos cadastrados. Sendo que 1.338 são acompanhados pelas ESF, porém quando se trata de atendimentos, apenas 891 hipertensos foram atendidos em 2015 (BRASIL, 2015).

Esta pesquisa contou com uma amostra de 20 pessoas diagnosticadas com Hipertensão Arterial, usuários da Unidade Básica de Saúde, maiores de 18 anos e de ambos os sexos. Adotou-se como critérios de inclusão para participação na pesquisa aqueles que estavam cadastrados na UBS, que foi inclusa para este estudo, e que tenham realizado pelo menos duas consultas para o acompanhamento da hipertensão; por considerar como um prazo adequado para o paciente identificar o tipo de serviço prestado na unidade. $\mathrm{O}$ tempo de realização das consultas varia de quarenta minutos a uma hora. Foram excluídos os pacientes que apresentavam hipertensão e diabetes conjuntamente, visto que o estudo buscou focar apenas o atendimento aos hipertensos. 
O contato com os hipertensos ocorreu em seus domicílios, após identificação da área, com maior número de hipertensos pela agente comunitária de saúde. A entrevista compreendeu questões relacionadas ao perfil socioeconômico, ao conhecimento que a população participante tem sobre o acolhimento, a interação da clientela com os serviços de saúde, bem como o conhecimento acerca da assistência prestada.

$\mathrm{Na}$ entrevista, ocorreu a gravação das falas e posteriormente, as transcrições das mesmas garantindo a fidedignidade. As entrevistas ocorreram em pelo menos cinco visitas em dias alternados, foram escolhidos os dias em que havia disponibilidade para a pesquisadora e a agente comunitária de saúde. A coleta foi realizada no mês de outubro de 2016.

A análise dos dados ocorreu por meio do Discurso do Sujeito Coletivo (DSC). Os DSC advindos da análise das falas foram discutidos por meio da literatura acerca da temática. Para análise, utilizou-se três das figuras metodológicas que foram as ideias centrais, as expressões chaves e que foram agrupadas para originar o discurso do sujeito coletivo. As figuras metodológicas foram estruturadas em tabelas utilizando a Microsoft Word 2010.

A pesquisa foi desenvolvida obedecendo a todos os critérios da Resolução $\mathrm{N}^{\circ} 466$, de 12 de dezembro de 2012 (BRASIL, 2012), e só foi realizada após o consentimento da Secretaria Municipal de Saúde mediante assinatura da declaração de anuência. Aos indivíduos que aceitaram espontaneamente participar do estudo, foram esclarecidos sobre a pesquisa, seus objetivos e métodos, à forma de participação, à garantia do anonimato. Após o esclarecimento no Termo de Consentimento Livre e Esclarecido, solicitou-se sua assinatura no Termo de Consentimento Pós-esclarecido, que garantia o sigilo de suas informações pessoais e o direito de desistir da pesquisa no momento que achasse oportuno. $\mathrm{O}$ presente estudo foi aprovado pelo Comitê de Ética em Pesquisa da Universidade Regional do Cariri pelo parecer de $\mathrm{n}^{\circ}$ 1.937.176.

\section{Resultados e Discussão}

Diante dos dados analisados constatou-se que o sexo feminino prevalece entre os usuários da unidade de saúde, representando quinze (75\%) dos entrevistados, com apenas cinco (25\%) desses entrevistados sendo do gênero masculino. A faixa etária que apresentou maior prevalência foi a entre 61 a 70 anos correspondendo a $35 \%(f=07)$, seguida pelos de 50 a 60 anos com $30 \%(f=06)$ e aqueles que se encaixavam acima dos 71 anos representaram $30 \%$ dos 
entrevistados $(f=06)$. Quanto à escolaridade, seis (30\%) dos entrevistados nunca estudaram, enquanto que dez $(50 \%)$ dos indivíduos possuem o ensino fundamental incompleto caracterizando como o maior índice, três (15\%) dos entrevistados concluíram o ensino fundamental e um (5\%) o ensino médio completo. A caracterização do perfil de acordo com o estado civil mostra que a maioria dos participantes são casados dez (50\%), seguidos pelos viúvos (as) sete (35\%), e que apenas dois (10\%) mantém relacionamento estável e um (5\%) é solteiro (a). Na variável ocupação profissional, evidenciou-se que um (5\%) dos entrevistados tem como profissão a agricultura, 14 (70\%) são aposentados, três (15\%) beneficiários e duas (10\%) donas de casa. Isso se relaciona diretamente com a renda dos mesmos, em que 19 (95\%) recebem entre um e dois salários mínimos, e apenas um (5\%) possuem menos de um salário.

Tabela 1 - Perfil Sociodemográfico dos participantes

\begin{tabular}{|c|c|c|}
\hline Sexo & $F$ & $\%$ \\
\hline Feminino & 15 & $75 \%$ \\
\hline Masculino & 05 & $25 \%$ \\
\hline Total & & $100 \%$ \\
\hline Idade & $F$ & $\%$ \\
\hline $20-40$ anos & 01 & $5 \%$ \\
\hline $50-60$ anos & 06 & $30 \%$ \\
\hline $61-70$ anos & 07 & $35 \%$ \\
\hline$>71$ anos & 06 & $30 \%$ \\
\hline Escolaridade & $F$ & $\%$ \\
\hline Analfabetos & 06 & $30 \%$ \\
\hline Ensino Fundamental Incompleto & 05 & $50 \%$ \\
\hline Ensino Fundamental Completo & 03 & $15 \%$ \\
\hline Ensino Médio Completo & 01 & $5 \%$ \\
\hline Total & & $100 \%$ \\
\hline Estado Civil & $F$ & $\%$ \\
\hline Casados & 10 & $50 \%$ \\
\hline Viúvos & 07 & 35 \\
\hline Relacionamento Estável & 02 & $10 \%$ \\
\hline Solteiro & 01 & $5 \%$ \\
\hline Total & & $100 \%$ \\
\hline Ocupação Profissional & $F$ & $\%$ \\
\hline Agricultor & 01 & $5 \%$ \\
\hline
\end{tabular}




\begin{tabular}{lll} 
Aposentado & 14 & $70 \%$ \\
Beneficiário & 03 & $15 \%$ \\
Donas de casa & 02 & $10 \%$ \\
Total & & $100 \%$ \\
Renda & $F$ & $\%$ \\
Maior que um salário mínimo & 01 & $5 \%$ \\
De um a dois salários mínimos & 19 & $95 \%$ \\
\hline
\end{tabular}

Fonte: Pesquisa direta, 2016.

Sobre o comparecimento na unidade de saúde, para o atendimento de suas necessidades, os usuários destacaram as dificuldades/facilidades percebidas acerca do acolhimento para este momento, conforme apresentado a seguir:

No Quadro 1, sobre quais os motivos que levam os participantes do estudo à consulta de hipertensão na atenção primária, os discursos apresentados se baseiam na renovação de receitas médicas para o recebimento da medicação (DSC 1) e/ou quando ocorre alguma intercorrência (DSC 2), vale ressaltar que alguns vão para a verificação da pressão arterial (DSC 3), mas nos seus discursos, fazem perceber a lacuna existente na assistência a esses pacientes pois percebeu-se que eles não referem a importância do acompanhamento pelos profissionais da saúde.

Quadro 1 - Comparecimento na unidade de saúde

\begin{tabular}{|c|c|}
\hline IDÉIA CENTRAL 1 & DSC 1 \\
\hline Renovação da receita & $\begin{array}{c}\text { "Só procuro o serviço de saúde a cada dois meses, quando preciso da } \\
\text { renovação da receita médica para pegar a medicação para a pressão" }\end{array}$ \\
\hline IDÉIA CENTRAL 2 & DSC 2 \\
\hline Sintomatologia aparente & "Eu só vou quando me sinto mal porque às vezes eu sinto muita \\
\hline IDÉIA CENTRAL 3 & sinto muito mal estar quando ela tá alta" \\
\hline Verificação da pressão & $\begin{array}{c}\text { DSC 3 } 3 \\
\text { ela tá e pegar a medicação, às vezes vou pra consulta mais é muito } \\
\text { difícil” }\end{array}$ \\
\hline
\end{tabular}

Fonte: Pesquisa direta, 2016. 
Diante do exposto pode-se levantar duas problemáticas: a falta de profissionais capacitados para realizar a educação em saúde, com os usuários do sistema de saúde e falhas no acolhimento desses pacientes, criando barreiras que impedem a criação de vínculos entre os usuários e os responsáveis pelo seu atendimento.

Motta et al. (2014) em estudo descritivo, qualitativo, afirmou mediante resultados de sua pesquisa, que a educação em saúde contribui diretamente para as mudanças na vida dos participantes tanto no que se refere a mudanças no hábito de vida como no conhecimento da doença, mudando a sua procedência na forma de agir.

O estudo de Azevedo (2014) sobre educação em saúde comprovou, por meio dos dados do Sistema de Informação da Atenção Básica de abril 2013, que após as ações de educação em saúde desenvolvidas, em uma unidade de atenção primária, os números de atendimento mensal aos hipertensos aumentaram, tendo como consequência o controle dos índices pressóricos dos hipertensos, como também conscientização dos mesmos quanto a Hipertensão Arterial Sistêmica.

Em relação ao acolhimento, em estudo realizado por Silva et al. (2013) com enfermeiros da ESF sobre o atendimento aos pacientes hipertensos, as falas dos sujeitos em uma das categorias trazem semelhanças com o discurso do sujeito coletivo 3 , demostrando claramente a quebra do vínculo entre os envolvidos no processo de saúde.

Apesar do presente estudo não ter sido realizado com enfermeiros da unidade de saúde, mas com os usuários hipertensos da unidade de saúde, encontrou-se pensamentos semelhantes. Os participantes do estudo não incluem como prioridade o acompanhamento profissional, em suas necessidades em saúde, que serão citadas adiante, seja por não terem o conhecimento da sua situação de saúde ou por acreditarem que apenas a medicação resolve a sua problemática.

Ao serem questionados se as suas necessidades em saúde eram atendidas, identificouse como produto final duas ideias centrais representadas no Quadro 2, representadas por bom atendimento e assistência ineficiente. Os entrevistados classificaram as suas necessidades apenas como sendo a aferição da pressão arterial e o recebimento de medicamentos, sendo assim eles relacionaram essas ações como decorrente de um bom atendimento. 
Quadro 2 - Necessidades em saúde

\begin{tabular}{|c|c|}
\hline IDÉIA CENTRAL 1 & DSC 1 \\
\hline Bom atendimento & "O atendimento é bom porque sempre verifico a pressão, recebo minha \\
medicação, resolvo tudo que tinha que fazer no posto"
\end{tabular}

Fonte: Pesquisa direta, 2016.

Na pesquisa realizada por Filha, Nogueira e Medina (2014) no município de Caxias MA desenvolvido com 1.024 usuários registrados no HiperDia, ao avaliar o trabalho dos profissionais da atenção primária sob a ótica dos usuários, evidenciou que $6,9 \%$ dos usuários classificaram como excelente, $64,1 \%$ descreveram como bom, 22,4\% como regular, 4,5\% relataram que o trabalho é ruim e 2,1\% referiram ser péssimo a assistência.

Tais dados é semelhante a esta pesquisa e permite afirmar que, é necessário compreender como os usuários avaliam o atendimento de suas necessidades em saúde, para melhora da assistência realizada.

Ainda sobre o mesmo tema, o estudo transversal baseado em dados da avaliação externa do Programa de Melhoria do Acesso e da Qualidade da Atenção Básica (PMAQ-AB), realizado em todo o território nacional brasileiro; um dos itens buscava a opinião do usuário quanto à qualidade do acolhimento ofertada na unidade de saúde e, 18,1\% dos usuários considerou como bom o atendimento, enquanto que para $9,6 \%$ era razoável e $0,7 \%$ consideravam como ruim; porém 33,6\% dos usuários não souberam responder (CLEMENTINO et al., 2015).

Percebe-se fragilidade dos usuários em avaliar o serviço no qual são atendidos. Pode-se supor que tal condição perpassa o desconhecimento, mas, especialmente, a falta de envolvimento dos usuários com a equipe de Saúde da Família (CLEMENTINO et al., 2015).

Pode-se perceber que os usuários limitam as suas necessidades prioritárias destacando apenas aquilo que lhe causa efeito imediato, o que pode causar dificuldades na interpretação da avaliação dos serviços ofertados. Vale destacar que, ao mesmo tempo em que, de acordo com as suas necessidades, o atendimento em saúde é considerado bom, esse mesmo atendimento 
demostra lacunas na assistência ofertando apenas uma parte mínima dos serviços que devem ser contemplados.

Quanto às facilidades e dificuldades encontradas pelos usuários hipertensos no serviço de saúde da atenção primária, como vemos no Quadro 3 e Quadro 4, percebe-se que esse tema abordado está interligado com o das necessidades em saúde, evidenciado no Quadro 2, pois o paciente ao considerar que tem um bom atendimento associa as suas necessidades em saúde solucionada, da mesma forma que as dificuldades são os fatores utilizados para classificar o atendimento de acordo com os depoimentos.

Quadro 3 - Dificuldades no atendimento

\begin{tabular}{|c|c|}
\hline IDÉIA CENTRAL 1 & DSC 1 \\
\hline Falta de Medicamentos & $\begin{array}{c}\text { "Encontram-se algumas dificuldades porque nem sempre tem a } \\
\text { medicação que eu uso, necessitando a compra dessa medicação" }\end{array}$ \\
\hline IDÉIA CENTRAL 2 & DSC 2 \\
\hline Atendimento prioritário & $\begin{array}{c}\text { "A única dificuldade é a espera na consulta, onde nem sempre os } \\
\text { idosos são prioridades passando várias horas na fila de espera e } \\
\text { também quando não tem médico na unidade" }\end{array}$ \\
\hline
\end{tabular}

Fonte: Pesquisa direta, 2016.

Quadro 4 - Facilidades no atendimento

\begin{tabular}{|l|c|}
\hline IDÉIA CENTRAL 1 & DSC 1 \\
\hline Bom atendimento & $\begin{array}{c}\text { "Até agora tudo tá acontecendo bem, o atendimento é muito bom, eu } \\
\text { sempre fico lá na fila e quando chega minha vez eu entro e sou } \\
\text { sempre bem atendida" }\end{array}$ \\
\hline
\end{tabular}

Fonte: Pesquisa direta, 2016.

A principal dificuldade encontrada trata-se da falta de medicamentos, que muitas das vezes não se têm nas unidades de distribuição, fazendo com que os pacientes tenham que comprar a medicação. Observa-se que o comparecimento nas consultas ocorre quase que unânime para a renovação da receita e que a ausência do profissional médico se torna uma dificuldade encontrada por esses usuários. 
Na maioria dos discursos, os participantes, referem-se ao profissional médico seja referente à falta desses profissionais e portanto; relatada como uma dificuldade no atendimento que repercute na resolução das suas necessidades, ou no principal motivo que os trazem a UBS para a renovação da receita pelo médico.

Para Clementino et al. (2015), o predomínio da consulta médica representa que ainda não ocorreu os avanços necessários da implantação do acolhimento na atenção primária, por ainda apresentar traços de um direcionamento único e que caso falhe; não há resolutividade dessa condição. Esse predomínio reproduz o modelo curativista em que o profissional da medicina se apresenta como figura central do processo saúde-doença, sendo a peça chave para resolutividade na atenção à saúde.

Por outro lado, quanto às facilidades no atendimento, os usuários afirmam que ocorre um bom atendimento por parte dos profissionais, mesmo referindo a espera na fila de demora em serem atendidos. Esse momento de espera torna apto para o desenvolvimento de ações voltadas a promoção e prevenção em saúde destinada a esses usuários.

Na representação do acolhimento (Quadro 5) buscou-se significar o que é acolhimento na visão dos pacientes, por entender-se que assim foi possível coletar a percepção desses pacientes sobre as categorias abordadas.

Ao serem questionados sobre como definiria acolhimento, o que se obteve fora dois tipos de discursos. O primeiro é de que não se tem o conhecimento acerca do assunto, que pode estar associado ao índice de escolaridade dos entrevistados. E no segundo discurso, encontrou se que o acolhimento é interpretado como o atendimento prestado na unidade, sendo assim o acolhimento depende da postura exercida pelos profissionais atuantes no serviço de saúde.

Quadro 5 - Significação do acolhimento

\begin{tabular}{|c|c|}
\hline IDÉIA CENTRAL 1 & DSC 1 \\
\hline Receber bem/ gentileza & $\begin{array}{c}\text { "É ser bem atendido na unidade de saúde, quando eles nos recebem bem. O } \\
\text { acolhimento é como se receber alguém na sua casa, você irá recebe-lo bem, } \\
\text { isto é gentileza" }\end{array}$ \\
\hline IDÉIA CENTRAL 2 & DSC 2 \\
\hline Incompreensão do tema & "Não sei o que é acolhimento" \\
\hline
\end{tabular}

Fonte: Pesquisa direta, 2016. 
Diante do exposto, entende-se que alguns pacientes não sabem definir o acolhimento. Isso foi identificado na ideia central 2, enquanto que outros associam ao fato de ser recebido bem, não tendo o conhecimento dos seus princípios como um todo. Porém, de acordo com Ferreira, Barreira e Sanches (2014) o acolhimento possui outras dimensões e dentro dessas inclui, atitudes de amparo e hospitalidade, que favorecem a construção de uma relação de confiança e compromissos dos usuários com as equipes e os serviços e contribui, assim, para a legitimação do sistema público.

Coutinho, Barbieri e Santos (2015) corroboram ao identificar que os usuários do serviço de saúde relacionam o acolhimento ao tratamento pessoal prestado pela equipe atuante e ao sistema estrutural, que ocorre desde o seu primeiro contato com o serviço, sendo assim, para eles atribui o mau atendimento a falta do acolhimento referente principalmente às filas de espera.

Os autores supracitados também relacionam o acolhimento à boa receptividade realizada pela equipe, o direcionamento para as consultas médicas, à dispensação de medicamentos, à triagem, e sobretudo, ao atendimento executado pelo profissional médico ou enfermeiro (COUTINHO; BARBIERI; SANTOS, 2015).

Santana et al. (2012) observaram que a percepção do que seria o acolhimento estava relacionada aos aspectos socioculturais e nível de instrução dos usuários, bem como as expectativas apresentadas no momento do atendimento para resolutividade de sua demanda.

\section{Considerações Finais}

O Acolhimento é uma fase do atendimento nos serviços de saúde que está ganhando repercussões na assistência, seja ela atenção primária ou hospitalar e, portanto, ele não permite a banalização do seu significado.

Quanto às dificuldades percebidas, à espera pelo atendimento e a falta de medicamentos; destacaram-se como os mais relevantes de a serem sanados para melhoria da assistência e consequente, interação entre os usuários e profissionais da saúde e que pode favorecer o vínculo entre os envolvidos.

Para que essas dificuldades venham a ser solucionadas ou minimizadas, é necessário que a equipe de saúde recorra às tecnologias leves, que incluem o acolhimento e também 
adotem medidas de educação permanente para a comunidade. Uma das estratégias, que podem ser adotada para que os pacientes percebam o acolhimento recebido é a educação em saúde.

Sendo assim, contata-se que as ações de educação em saúde são adequadas para poder satisfazer as necessidades de saúde da população, preservando a sua autonomia, valorizando o seu saber e buscando uma melhoria na sua qualidade de vida. E essas ações voltadas ao acolhimento podem modificar a concepção dos usuários quanto ao atendimento.

\section{Referências}

AZEVEDO, L.M.A.P.; CABRAL, S.A.L. Educação em Hipertensão Arterial na Estratégia Saúde da Família Dona Heloisa, Brasília de Minas - Minas Gerais. Montes Claros/Minas Gerais. 2014. 42 p. Monografia (especialização) - Universidade Federal de Minas Gerais. 2014.

BARRETO, M.S.; SILVA, R.L.D.T.; WAIDMAN, M.A.P.; MARCON, S.S. A Trajetória das Políticas Públicas de Saúde para Hipertensão Arterial Sistêmica no Brasil. Esc Anna Nery. v. 20, n. $01, \quad$ p. 460-468, 2013. Disponível em: https://aps.ufjf.emnuvens.com.br/aps/article/view/1928/770

BRASIL. Ministério da Saúde. Ambiência. Brasília: Ministério da Saúde, 2013.

BRASIL. Ministério Da Saúde. Política Nacional de Atenção Básica. Portaria No 2.436, de 21 de setembro de 2017 Aprova a Política Nacional de Atenção Básica, estabelecendo a revisão de diretrizes para a organização da Atenção Básica, no âmbito do Sistema Único de Saúde (SUS). Brasília - DF. 2017.

BRASIL. Ministério da Saúde. Sistema de Informação de Atenção Básica. Brasília-DF. 2015.

BRASIL. Resolução no 466, de 12 de dezembro de 2012. Diário oficial da União, no 12.

CLEMENTINO, F.S.; GOMES, L.B.; VIANNA, R.P.T.; MARCOLINO, E.C.; ARAÚJO, J.P.; CHAVES, T.V. Acolhimento na Atenção Básica: Análise a partir da Avaliação Externa do Programa de Melhoria do Acesso e da Qualidade da Atenção Básica (PMAQ-AB). RSC Online. v. 4, n. 1, p. 62-80, 2015. Disponível em: http://www.ufcg.edu.br/revistasaudeeciencia /index.php/RSC-UFCG/article/view/229/160

COUTINHO, L.R.P.; BARBIERI, A.R.; SANTOS, M.L.M. Acolhimento na Atenção Primária à Saúde: revisão integrativa. Saúde Debate [online]. v. 39, n. 105, p. 514-524, 2015. DOI: https://doi.org/10.1590/0103-110420151050002018

FERREIRA, M.L.S.M.; BARREIRA, P.R.M.; SANCHES, M.M.J. Acolhimento na Percepção dos enfermeiros da Atenção Primária à Saúde. Rev Aquichan. v. 14, n, 2, p. 216-225, 2014. Disponível em: https://repositorio.unesp.br/handle/11449/136914 
FILHA, F.S.S.C.; NOGUEIRA, L.T.; MEDINA, M.G. Avaliação do controle de hipertensão e diabetes na Atenção Básica: perspectiva de profissionais e usuários. Saúde Debate [online]. v. 38, n. SPE, p. 265-278, 2014. Disponível em: http://www.redalyc.org/pdf/4063/406341750020.pdf

GUIMARÃES, J.M.; FREITAS, G.H.; ARAÚJO, A.F.; FREITAS LIMA, M.C.; MACHADO, É.B.; CARNEIRO, C. et al. Care Management In The Family Health Support Core: Technologies Operated In The Professional Dimension. Int Arch Med. v. 10, n. 92, 2017.

LIMA, L.L.; MOREIRA, T.M.M.; JORGE, M.S.B. Produção do cuidado a pessoas com hipertensão arterial: acolhimento, vínculo e corresponsabilização. Rev Bras Enferm. v. 66, n. 4, p. 514-522, 2013. DOI: http://dx.doi.org/10.1590/S0034-71672013000400008

MALACHIAS, M.V.B.; SOUZA, W.K.S.B.; PLAVNIK, F.L.; RODRIGUES, C.I.S.; BRANDÃO, A.A.; NEVES, M.F.T. et al. $7^{\mathrm{a}}$ Diretriz Brasileira de Hipertensão Arterial. Arq Bras Cardiol. v. 107, n. 3, p. 1-83, 2016. Disponível em: http://www.scielo.br/scielo.php?script=sci_arttext\&pid=S0066-782X2010001700001

MOTTA, M.D.C.; PETERNELLA, F.M.N.; SANTOS, A.L.; TESTON, E.F.; MARCON, S.S. Educação em Saúde Junto a Idosos com Hipertensão e Diabetes: Estudo Descritivo. Revista Uningá Review. $\quad$ v. 18, n. 2, p. 48-53, 2014. Disponível em: https://www.mastereditora.com.br/periodico/20140501_121328.pdf

OLIVEIRA, J.R.; ALBUQUERQUE, M.C.S.; BRÊDA, M.Z.; BARROS, L.A.; LISBÔA, G.L.P. Concepções e Práticas de Acolhimento Apresentadas pela Enfermagem no Contexto da Atenção Básica à Saúde. Rev Enferm UFPE Online. v. 9, n. 5, p. 1545-1555, 2015. Disponível em: https://periodicos.ufpe.br/revistas/revistaenfermagem/article/view/10869/12104

OLIVEIRA, M.A.C.; PEREIRA, I.C. Atributos essenciais da Atenção Primária e a Estratégia Saúde da Família. Rev Bras Enferm. v. 66, n. spe, p. 158-164, 2013. DOI: http://dx.doi.org/10.1590/S0034-71672013000700020

OLOWE, O.; ROSS, A. Knowledge, adherence and control among patients with hypertension attending a peri-urban primary health care clinic, KwaZulu-Natal. Afr J Prim Health Care Fam Med. v. 9, n. 1, p. 1456, 2017. Disponível em: http://www.phcfm.org./index.php/phcfm/article/view/1456

SANTANA, J.C.; FORTES, N.M.; ANDRADE, A.V.; SOARES, A.P.F.; LIMA, J.R.M. Acolhimento em um serviço da Atenção Básica à Saúde de Minas Gerais. Rev Enferm Cent.Oeste Min. v. 2, n. 2, p. 166-176, 2012. Disponível em: http://www.seer.ufsj.edu.br/index.php/recom/article/view/199

SANTOS, F.P.A.; NERY, A.A.; MATUMOTO, A.S. A produção do cuidado a usuários com hipertensão arterial e as tecnologias em saúde. Rev Esc Enferm. USP. v. 47, n. 1, p. 107-114, 2013. DOI: http://dx.doi.org/10.1590/S0080-62342013000100014 
SILVA, F.V.F.; SILVA, L.F.; GUEDES, M.V.C.; MOREIRA, T.M.M.; RABELO, A.C.S.; PONTE, K.M.A. Cuidado de Enfermagem a pessoas com hipertensão fundamentado na teoria de parse. Esc Anna Nery. v. 17, n. 1, p. 111-119, 2013. Disponível em: http://www.scielo.br/pdf/ean/v17n1/16.pdf

\section{Como citar este artigo (Formato ABNT):}

SILVA FILHO, José Adelmo da; CUNHA, Milana Correia; SANTOS, Rosely L. dos; VICTOR, Itala Alencar B.; VIEIRA, Roberta P.; OLIVEIRA, Mikaela M. de. Percepção dos Pacientes Hipertensos sobre o Acolhimento na Atenção Primária à Saúde. Id on Line Revista Multidisciplinar e de Psicologia, 2018, vol.12, n.40, p.980-994. ISSN: 1981-1179.

Recebido: 21.05.2018

Aceito: 28.05.2018 